\title{
SEGURANÇA DO PACIENTE NA PREVENÇÃO DE LESÃO POR PRESSÃO EM TEMPOS DE PANDEMIA: RELATO DE EXPERIÊNCIA
}

\author{
PATIENT SAFETY IN PREVENTING PRESSURE INJURY IN PANDEMIC TIMES: \\ EXPERIENCE REPORT
}

\author{
Flávia Martins Branco ${ }^{1} *$ Dayane Martins da Silva Campos $^{2} *$ Eva Natalina Ferreira Costa $^{3}$
}

\section{RESUMO}

Objetivo: apresentar os métodos utilizados para promover a prevenção de ocorrência de Lesão por Pressão (LPP) durante quatro meses de atuação no setor clínica médica. Metodologia: O presente estudo descritivo de abordagem qualitativa, do tipo relato de experiência, durante nossa atividade como enfermeiras residentes no setor clínica médica de um Hospital Federal na cidade do Rio de Janeiro, no período do curso de Pós-Graduação em nível de especialização em Clínica Médica e Cirúrgica, sob a forma de treinamento em serviço, no período de julho a outubro do ano de 2020. Resultados: o estudo evidenciou que o dimensionamento pessoal da equipe de Enfermagem afeta diretamente a incidência e prevalência da LPP. O estudo reforça que a aplicação da Escala de Risco de Braden permite a tomada de decisão e direcionamento das intervenções de enfermagem para cada paciente. Considerações Finais: é essencial a interação com protocolos e realizar ajustes nas ações previstas que têm impacto ou ação direta no comprometimento da pele do paciente. Entende se como fator importante para a confecção do curativo ideal com cobertura adequada, contudo torna-se primordial a manutenção da prevenção das lesões por pressão. Assim fazem os enfermeiros e suas equipes da Unidade de Internação Clínica desta Instituição.

Palavras-chave: Segurança do Paciente; Lesão por Pressão; Enfermagem; Prevenção de Doenças; Enfermeiras e Enfermeiros.

\begin{abstract}
Objective: to present the methods used to promote the prevention of the occurrence of Pressure Injury (PPL) during four months of work in the medical clinic sector. Methodology: This is a descriptive study of a qualitative approach, of the experience report type, during our activity as resident nurses in the clinical medicine sector of a Federal Hospital in the city of Rio de Janeiro, during the Post-Graduate course at the level of specialization in Medical and Surgical Clinic, in the form of in-service training, from July to October of 2020. Results: the study showed that the personal dimension of the Nursing team directly affected an increase and prevalence of LPP. The study reinforces that the application of the Braden Risk Scale allows decision-making and targeting of nursing therapies for each patient. Final Considerations: it is essential to interact with protocols and make adjustments to predefined actions that impact or directly affect the patient's skin. It is understood as an important factor for the configuration of the ideal dressing with adequate coverage, it is essential to maintain the prevention of pressure injuries. So do the nurses and their teams of the Clinical Inpatient Unit of this institution.

Keywords: Patient Safety; Pressure Ulcer; Nursing; Disease Prevention; Nurses.

\footnotetext{
${ }^{1}$ Residente de Enfermagem pela Universidade Federal do Estado do Rio de Janeiro (UNIRIO). E-mail: $\underline{\text { flaviabrancoenf@gmail.com }}$ ORCID: https://orcid.org/0000-0003-3484-8555

${ }^{2}$ Residente de Enfermagem pela Universidade Federal do Estado do Rio de Janeiro (UNIRIO). E-mail: campos dayane@ hotmail.com ORCID: https://orcid.org/0000-0002-0193-4417

${ }^{3}$ Residente de Enfermagem pela Universidade Federal do Estado do Rio de Janeiro (UNIRIO). E-mail: evacostaferreira@gmail.com ORCID: https://orcid.org/0000-0003-2673-6967
} 


\section{INTRODUÇÃO}

A Lesão por Pressão (LPP) é caracterizada por um ferimento da pele ou em algum tecido subjacente, ocasionado sobre uma proeminência óssea, em consequência da pressão continua ou de uma associação entre esta e a força de torção. As LPP também estão agregadas a vários aspectos contribuintes, nos quais ainda não se tem um completo esclarecimento ${ }^{1}$.

A Associação Brasileira de Estomoterapia (SOBEST) refere que, as LPP podem ser classificadas em 4 estágios: 1 - pele íntegra com eritema, que se realizado uma pressão digital, não embranquece. 2 - perda parcial da pele com exposição da camada derme, podendo ter a presença de bolhas. 3 - perda da pele com espessura total da epiderme e presença de tecido subcutâneo. 4 - perda da pele com espessura total e exposição de músculos, tendões, ligamentos e ossos ${ }^{2}$.

O National Pressure Ulcer Advisory Panel (NPUAP), entre os dias de 8 e 9 de abril de 2016, realizou atualizações nas terminologias associadas as Úlceras por Pressão (UPP), modificando para Lesões por Pressões, essa mudança ocorreu devido ao fator de que no $1^{\circ}$ estágio não se encontra uma úlcera, sendo assim, o termo caiu em desuso. Além disso, adicionaram o termo lesão por pressão relacionada a dispositivo médico, descrevendo qualquer lesão relacionada a dispositivos e/ou equipamentos utilizados na área da saúde ${ }^{2}$.

No ano de 2013, foi instituído pelo Ministério da Saúde (MS), o Programa Nacional de Segurança do Paciente (PNSP). O programa possui como objetivo contribuir para uma melhor qualidade na assistência prestada ao indivíduo, em todo território nacional, em qualquer unidade de saúde. Para alcançar esse objetivo, estabeleceu-se 6 metas de Segurança do Paciente, entre essas metas inclui-se a redução dos riscos de quedas e de lesões por pressões $^{3}$.

Um estudo de Malagutti e Kahihara ${ }^{4}$, realizado em um Hospital Universitário, demonstrou uma incidência de 39,81\% para as LPP. As taxas apresentadas de incidência e prevalência, apresentam alterações que se devem as particularidades dos pacientes e do seu nível de assistência, diferenciando em cuidados agudos com incidência de 0,4 a $38 \%$, prevalência de 10 a $18 \%{ }^{1}$.

A prescrição e avaliação dos cuidados com a pele do paciente é uma competência do enfermeiro, sendo que a participação da equipe interdisciplinar na prevenção e tratamento das alterações apresentadas é extremamente importante para a contribuição e planejamento da assistência prestada ao indivíduo em risco ${ }^{5}$.

A equipe de enfermagem, por contemplar o grupo mais numeroso da equipe de saúde, tem 
um papel fundamental para garantir a segurança do cuidado desses pacientes com lesões. Ademais, são esses profissionais que atuam diretamente com o paciente sendo responsáveis por prevenir agravos e incidentes ${ }^{6}$.

Um dos fatores extrínsecos que impactam na prevenção de lesão por pressão é o dimensionamento de enfermagem, tendo em vista que a qualidade dos cuidados prestados aos pacientes está diretamente proporcional ao quantitativo de pessoal de enfermagem. Assim como disposto na Resolução do COFEN $\mathrm{N}^{\circ} 543 / 2017$ os quantitativos e qualitativos de profissionais de enfermagem interferem, diretamente, na segurança e na qualidade da assistência ${ }^{7}$.

Este estudo tem como objetivo apresentar os métodos utilizados para promover a prevenção de ocorrência de Lesão por Pressão (LPP) durante quatro meses de atuação no setor Unidade de Internação Clínica, tendo como questão norteadora: Quais as estratégias e os cuidados de enfermagem ofertados aos pacientes que apresentam lesão por pressão em Unidade de Internação Clínica durante a pandemia da COVID-19?

\section{METODOLOGIA}

Trata-se de um estudo descritivo de abordagem qualitativa, do tipo Relato de Experiência. O Relato de Experiência define-se como uma construção aberta a considerações, estimulando novos estudos, através de vivências, histórias e conhecimentos ${ }^{8}$.

O curso de pós-graduação em nível de especialização, sob a forma de treinamento em serviço para Enfermeiro, nos moldes de residência visa um preparo com base científica, viabilizando aos enfermeiros uma competência técnica e julgamento clínico diante dos cuidados prestados ao indivíduo.

$\mathrm{O}$ relato de experiência foi descrito durante nossa atividade como enfermeiras residentes no setor clínica médica de um Hospital Federal na cidade do Rio de Janeiro, no período do curso de Pós-Graduação em nível de especialização em Clínica Médica e Cirúrgica sob a forma de treinamento em serviço, no período de julho a outubro do ano de 2020.

Para obtenção das informações, utilizou-se das vivências, da realização da Escala de Braden em todos os pacientes internados e das planilhas de dados implementada pelo próprio setor da instituição, que continha as informações mensais dos pacientes internados.

\section{RELATO DE EXPERIÊNCIA}

A Instituição tem uma emergência com "porta aberta", sendo também uma referência para tratamentos oncológicos, então sofreu 
grande impacto em decorrência do COVID 19.

No final do mês de março de 2020, aumentou-se drasticamente o número de pacientes internados, devido a pandemia da COVID 19, sendo necessário a criação e separação de uma ala no setor clínica médica aos pacientes diagnosticados com coronavírus.

Durante os 4 meses de atuação dentro do setor Unidade de Internação Clínica, objetivou-se assegurar a prevenção de eventos adversos relacionados a assistência de saúde. Dentre esses eventos adversos, destaca-se a prevenção de LPP, essa ênfase tem-se relação com a pandemia, pois surgiram muitos motivos que intensificaram o desenvolvessem dessas lesões.

Outro fator importante que ocorreu durante o período da pandemia, foi o menor dimensionamento de pessoal, devido a quantidade de pacientes internados. Inclui-se ainda, os profissionais que tiraram licenças no período da pandemia, por serem considerados grupos de risco e muitos dos que mantiveramse trabalhando se infectaram pelo vírus, sendo necessário o isolamento por 15 dias ou enquanto manifestava sinais e sintomas da doença. Todos esses fatores impactaram diretamente na assistência à saúde, pois eram poucos profissionais para a prática do cuidado voltado para o desenvolvimento de lesões, como a realização da mudança de débito, que com a diminuição dos profissionais e dos acompanhantes, passou a ser uma prática que carecia de muito tempo.

Em decorrência disso, o olhar da equipe interdisciplinar durante este processo foi extremamente importante para o sucesso desta ação, sendo possível visualizar que apesar do grande número de pacientes com LPP no setor, quase $100 \%$ foram desenvolvidas previamente em outros ambientes, durante estes 4 meses apenas uma paciente desenvolveu uma LPP dentro do setor, uma paciente obesa, em paliação, com fatores nutricionais comprometidos, baixa perfusão, comorbidades e fatores sociais comprometidos. Essa informação confirma a importância de cuidados de toda equipe para essas lesões, buscando a menor incidência possível.

A avaliação das lesões feitas de forma adequada e a decisão da cobertura a ser utilizada em cada caso, possuem grande relevância neste processo. $\mathrm{Na}$ instituição existe uma Comissão de Tratamento e Prevenção de Feridas, com a função de auxiliar nos assuntos relacionados a feridas e lesões, auxiliando na avaliação e escolha da cobertura adequada para cada caso.

Para avalição do risco de desenvolvimento de lesões nesses pacientes, utilizou-se a escala de Braden em todos os pacientes internados no setor, possibilitando a 
aplicação de medidas preventivas.

Durante a pandemia surgiu-se um novo grande desafio, as lesões ocasionadas pelas máscaras n95 e PFF2 nos profissionais de saúde, pois não é possível fazer a substituição do dispositivo/da máscara. Cita-se ainda as lesões acometidas por dispositivos médicos como cateter de oxigênio $\left(\mathrm{O}^{2}\right)$, nas quais realizavamse a troca de curativo com as coberturas indicadas pela comissão da própria Instituição e um rodízio dos locais de passagem e fixação do cateter, buscando evitar a pressão contínua na pele do paciente.

A avaliação diária foi fundamental para a qualidade do cuidado, sempre explicando para o paciente importância da alimentação, hidratação e da mudança de decúbito, buscando trabalhar inicialmente com a prevenção, evitando futuras LPP, pois trabalhar os métodos de prevenção é prestar uma assistência baseada em evidências científicas.

\section{RESULTADOS}

Para obtenção dos a seguir, utilizou-se planilhas de dados implementada pelo próprio setor, elaboradas através das informações mensais dos pacientes internados, identificados por números e sinalizações pertinentes ao tempo de permanência, grau de risco e acompanhamento pela equipe de enfermagem.

Figura 1 - Escala de Fugulin dos pacientes internados na clínica médica no mês de julho

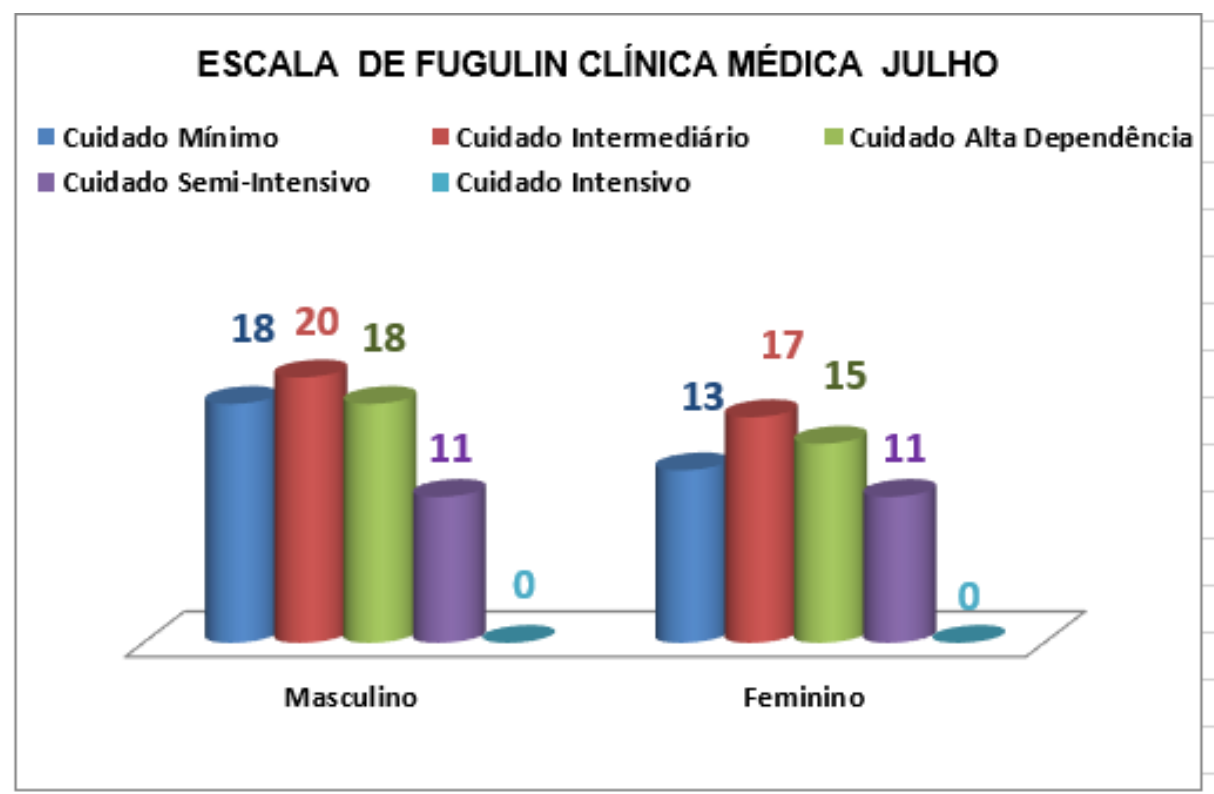

Fonte: Própria

Lesão por pressão é um evento adverso evitável e sua prevalência é considerada um marcador da característica dos cuidados de saúde. Neste gráfico pode-se observar que no 


\section{RELATOS}

mês em questão o setor teve 11 pacientes masculinos e 11 femininos com uma demanda de cuidados semi-intensivos, carecendo de maior atenção e cuidados dos profissionais, em resultado da necessitam de auxílio para realizar a mudança de decúbito, fator ressaltante para o desenvolvimento dessas lesões.

Figura 2 - Escala de Fugulin dos pacientes internados na clínica médica no mês de Agosto

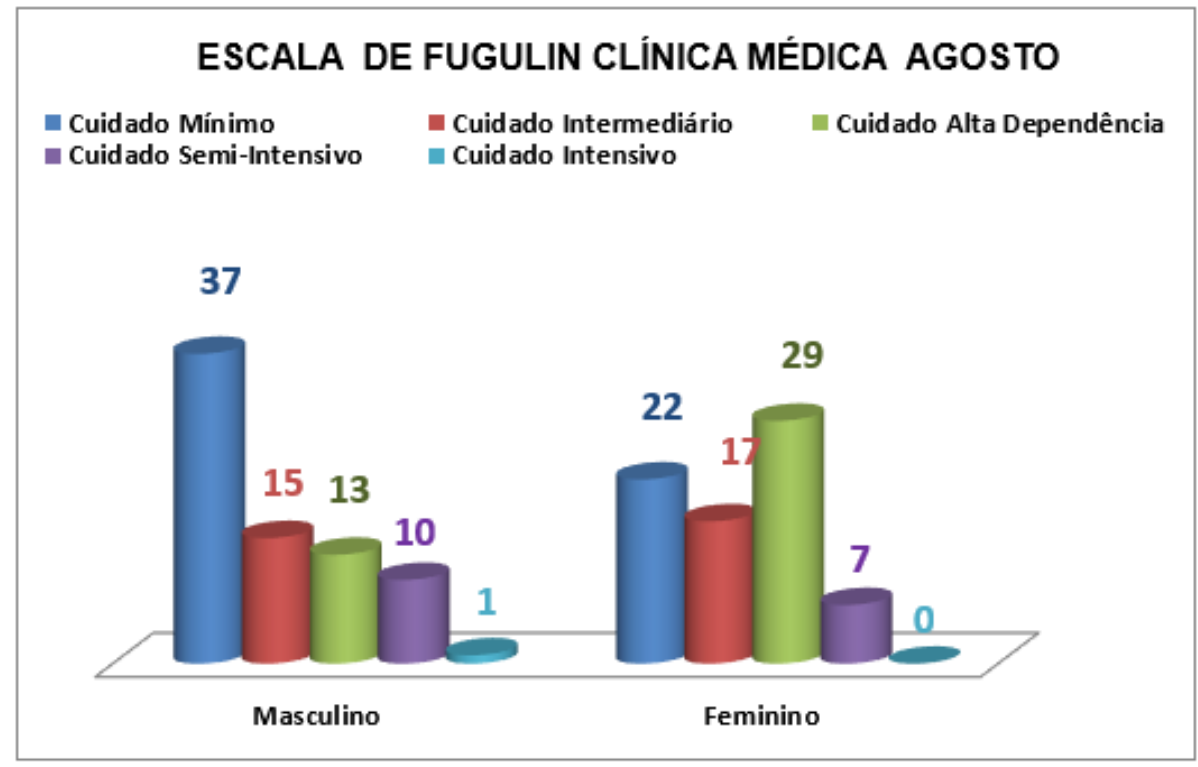

Fonte: Própria

O grau de dependência dos pacientes, é proporcional ao tempo estipulado para os cuidados dele, sendo assim, maiores graus de dependência requerem uma demanda maior de profissionais, o que pode provocar sobrecarga sobre os profissionais da saúde, com ênfase para os da Enfermagem, essa sobrecarga, acrescida as cargas horarias extensas podem provocar danos negativos na Segurança do Paciente. Neste gráfico podemos observar que neste mês tivemos 13 pacientes masculinos e 29 femininos que de acordo a classificação da escala necessitava de cuidados de alta dependência. 
Figura 3 - Escala de Fugulin dos pacientes internados na clínica médica no mês de Setembro

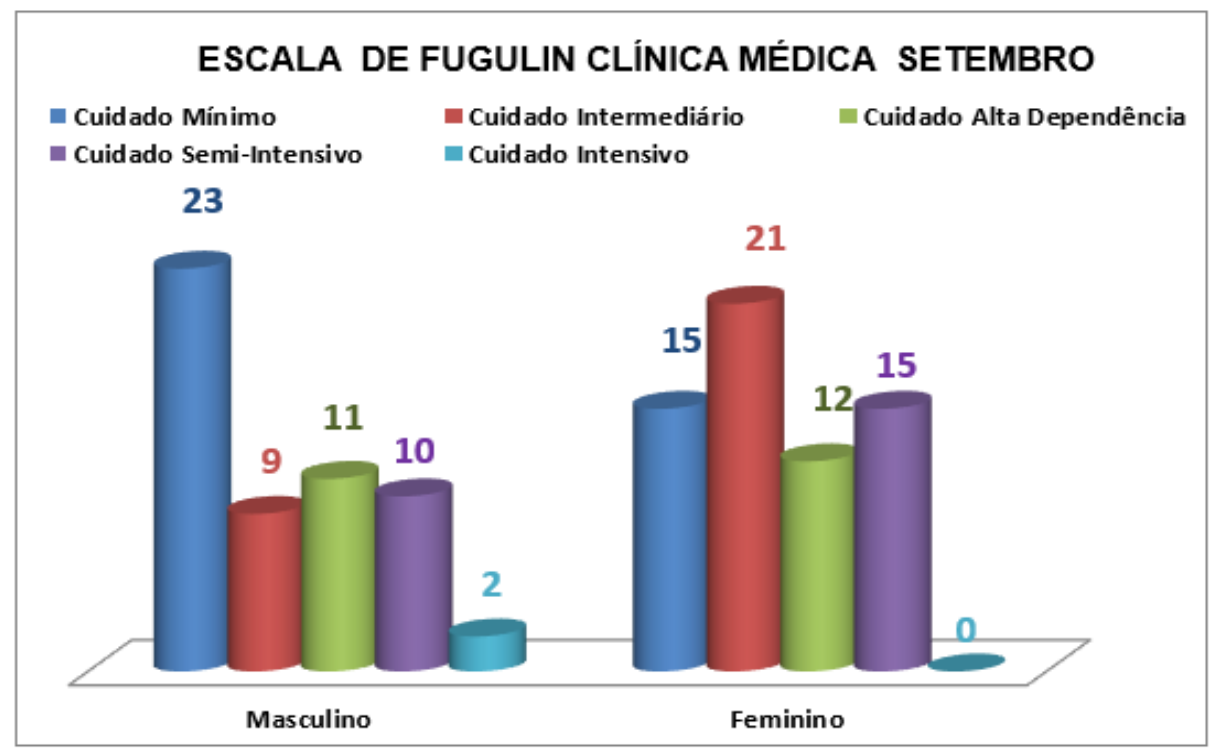

Fonte: Própria

A prevenção de lesões por pressão abrange intervenções relacionadas ao acompanhamento integral do paciente em risco de adquirir a lesão, por meio da utilização da escala de Fugulin, conseguimos um resultado positivo. Foi possível através da mesma designar os cuidados de acordo com a necessidade de cada usuário, principalmente se observarmos que sempre há prevalência de pacientes necessitando de cuidados intensivos e com alta dependência.

Figura 4 - Escala de Fugulin dos pacientes internados na clínica médica no mês de Outubro

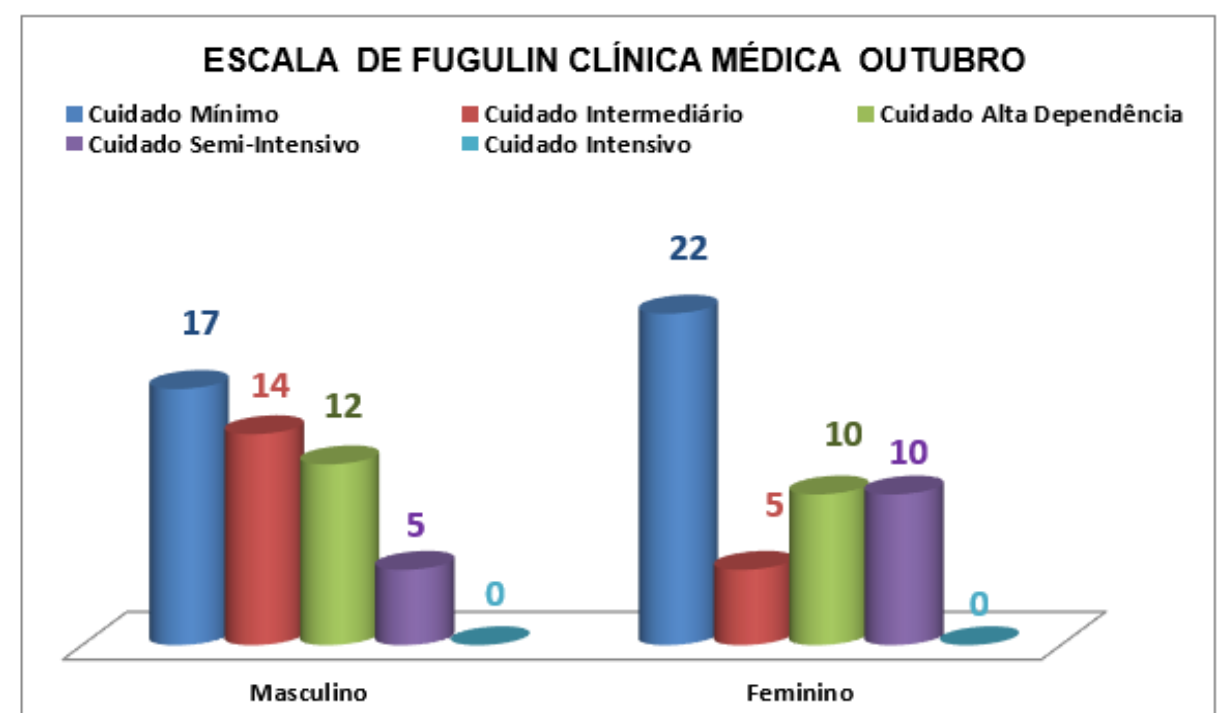

Fonte: Própria 
No que compete a prevenção de lesão por pressão, nota-se que os profissionais destinguem sua importancia e prestam cuidados fundamentados na ciência e em protolocolos institucionais, o que contribui para prática baseada em evidência, além de auxiliar no cuidado voltado para os pacientes que se encontram em tais necessidades, pois apesar da maioria estarem classificados em cuidados minimos, é de grande destaque a quantidade de usuarios que carecem de auxilio para suas atividades básicas da vida diária, de forma com que, sabe-se que paciente mais graves são em sua grande maioria restritos ao leito, sendo um fator importantissimo para a o desenvolvimento e o tratameto das lesões por pressões.

\section{DISCUSSÃO}

As lesões relacionadas aos dispositivos médicos são identificadas como lesão por pressão não classificadas, em consequência do fator de não ocorrerem em e sobre influência das regiões de proeminências ósseas, ela pode ocorrer em qualquer região do corpo, seja na pele ou mucosas que estejam sobre a pressão dos dispositivos médicos ${ }^{4}$.

Para avaliação do risco de desenvolvimento das lesões, destaca-se Escala de Braden, sendo essa, uma ferramenta excelente para a mensuração dos fatores indicativos para o desenvolvimento de uma
LPP, por meio do estabelecimento de pontuações baseados em uma série de parâmetro definidos. Essa escala é indicada pela Agência Nacional de Vigilância Sanitária para realizar a avaliação do paciente diariamente ou conforme protocolo do Hospital $^{1-9}$.

Os enfermeiros representam um papel essencial para a realização dessas ações, pois a enfermagem é uma ciência que possui como foco e essência a assistência e o cuidado, sendo a Escala de Braden um instrumento bastante utilizado por esses profissionais durante o trabalho, subsidiando seu cuidado, indicando provável complicação e apontando intervenções para prevenção do aparecimento de lesão por pressão $^{2-10}$.

No Brasil, as Instituições Hospitalares passaram a dar mais importância para a temática a partir da publicação da portaria 529, em abril do ano de 2013 pelo Ministério da Saúde, que institui o Programa Nacional de Segurança do Paciente, tendo como objetivo a diminuição, a um mínimo aceitável, dos eventos adversos associados a assistência de saúde. A portaria explica sua relação com as feridas, onde as lesões por pressão são consideradas um evento adverso, isto é, um incidente que origina um dano ao paciente. Devido a sua relevância, a ocorrência deste evento é de notificação compulsória mensal ${ }^{2}$. 
Os Índices de lesão por pressão internacionalmente, são considerados um relevante indicador de qualidade de cuidados de saúde. A organização Nacional de Acreditação e Joint Comission International, dirigente do processo de Acreditação Hospitalar, considera prevalência e incidência de LPP é indicada um considerável indicador de qualidade assistencial em enfermagem, autorizando analisar os fatos quanto a sua distribuição, os pacientes mais suscetíveis e as regiões em que são mais comuns o aparecimento das lesões ${ }^{7}$.

Segundo Silva et $\mathrm{al}^{11}$ "Tais lesões destacam-se como um relevante problema de saúde, uma vez que para o tratamento adequado, muitas vezes, os pacientes acometidos com lesões crônicas necessitem de permanência prolongada nas redes hospitalares".

É importante que o dimensionamento de pessoal de enfermagem, abrangido como um método que auxilia na antevisão dos recursos necessários para atender as necessidades de saúde, para que as Instituições de saúde possibilite, não só a utilização das escalas, como também a execução de ações preventivas para o desenvolvimento de lesão por pressão, como utilização de insumos adequados, mudança de decúbito com frequência, e equipe interdisciplinar envolvida no processo para proporcionar uma assistência diferenciada aos pacientes $^{3-12}$.

O quantitativo e o qualitativo de profissionais de enfermagem afeta, diretamente, na segurança e na qualidade da assistência prestada ao paciente, além de proporcionar desafios no atendimento a todas as demandas ${ }^{6-}$ 13.

\section{CONSIDERAÇÕES FINAIS}

O estudo evidencia uma baixa incidência de lesão por pressão nos pacientes internados na Unidade de Internação Clínica neste período, decorrente das práticas de cuidado e da participação de uma comissão especifica sobre a temática em questão, levando-se em consideração a não inclusão dos usuários com lesões provenientes de outras unidades e outros setores.

A literatura nacional ainda é escassa sobre a incidência e prevalência de LPP em Instituições Brasileiras, sendo fundamental o reconhecimento desse indicador em cada instituição, para a elaboração condutas especificas perante a necessidade de cada local, objetivando à Sistematização da Assistência de Enfermagem a partir dos resultados encontrados.

O estudo reforça que a aplicação da Escala de Risco de Braden no ato da admissão permite a tomada de decisão e direcionamento das intervenções de enfermagem para cada 
paciente, o mais precocemente, sendo importante também avaliar o nível de adesão para prevenção de LPP de todos os profissionais e não somente da Enfermagem.

Visando melhoria da assistência a Comissão de Prevenção e Tratamentos de Feridas (CPTF) da Instituição realiza avaliações e planejam condutas por Enfermeiras especialistas e o risco de desenvolvimento de lesão por pressão deve ser reavaliado diariamente por toda a equipe.

Assim é essencial a interação com protocolos e fazer ajustes nas ações previstas que têm impacto ou ação direta no comprometimento da pele do paciente. Porque mais importante que realizar o curativo com a cobertura adequada é trabalhar com as medidas preventivas das devidas lesões.

Acredita-se que este trabalho traga contribuições e incentivos de boas práticas para a equipe. E que continuem revisando seus processos gerenciais e assistenciais, garantindo a segurança e qualidade dos serviços prestados aos pacientes.

\section{REFERÊNCIAS}

1 National Pressure Ulcer Advisory Panel (NPUAP). National Pressure Ulcer Advisory Panel (NPUAP) announces a change in terminology from pressure ulcer to pressure injury and updates the stages of pressure injury, Washington: NPUAP; 2019. https://www.sobenfee.org.br/artigos acesso em:
7 nov. 2020.

2 Associação Brasileira de Estomaterapia SOBEST e da Associação Brasileira de Enfermagem em Dermatologia- SOBENDE, 2016 . http://www.sobest.org.br/textod/35. Acesso em: 06 nov. 2020.

3 Nota Técnica GVIMS/ GGTES n. 03/2017. Práticas seguras para prevenção de Lesão por Pressão em serviços de saúde, Agência Nacional de Vigilância Sanitária (ANVISA). https://www20.anvisa.gov.br/segurancadopacie nte/index.php/alertas/item/nota-tecnica-gvimsggtes-03-2017. Acesso em: 06 nov. 2020.

4 Malagutti W, Kahihara CT. Curativos, estomias e dermatologia: uma abordagem multiprofissional. 3. ed. São Paulo: Martinari; 2014. $640 \mathrm{p}$.

5 Ministério da Saúde. Anvisa. Fiocruz Protocolos para prevenção de úlcera por pressão. 09/07/2013. Disponível em: https://www20.anvisa.gov.br/segurancadopacie nte/index.php/publicacoes/item/ulcera-porpressao. Acesso em: 14 nov. 2020

6 Busanello, Josefine et al. Cuidados de Enfermagem ao Paciente adulto: Prevenção de Lesões Cutaneomucosas e Segurança do Paciente. Revista de Enfermagem Reufsm, Santa Maria, p. 597-606, 04 dez. 2015. Trimestral. Disponível em: https://pesquisa.bvsalud.org/portal/resource/pt/b de-27658. Acesso em: 08 nov. 2020.

7 Conselho Federal de Enfermagem-COFEN. Resolução 543/2017. Atualiza e estabelece parâmetros para dimensionamento do quadro de profissionais de Enfermagem nos serviços/locais em que são realizadas atividades de enfermagem. Disponível em: http://www.cofen.gov.br/resolucao-cofen5432017_51440.html. Acesso em: 15 nov. 2020.

8 Daltro MR, Amélia A. Relato de experiência: 
Uma narrativa científica na pós-modernidade. Estudos e Pesquisas em Psicologia [Internet]. 2019 [cited 2021 May 6];19(1):223-37. Available from: https://www.epublicacoes.uerj.br/index.php/revispsi/article/vi ew/43015/29664. Acesso em: 15 nov. 2020.

9 Borghardt AT, Prado TN. do, Araújo TM de, Rogenski NMB, Bringuente ME de O. Avaliação das escalas de risco para úlcera por pressão em pacientes críticos: uma coorte prospectiva . Revista Latino-Americana De Enfermagem, 2015;23(1):28-35. Disponível em: https://doi.org/10.1590/0104-1169.0144.2521. Acesso em: 15 nov. 2020.

10 Jansen RCS, Silva KB de A, Moura MES. Braden Scale in pressure ulcer risk assessment. Revista Brasileira de Enfermagem [Internet]. 2020 [cited 2021 May 7];73(6). Available from: https://www.scielo.br/scielo.php?pid=S0034$71672020000600169 \&$ script=sci_arttext\&tlng=

Submissão: 2021-06-16

Aprovado: 2021-07-13

\section{Autor correspondente}

Eva Natalina Ferreira Costa, Rua Santo Amaro184, Apto 307, CEP 22211.230, +55 21 993130672 evacostaferreira@gmail.com.

Fomento e Agradecimento: A toda equipe de Enfermagem da Unidade de Internação Clínica, Comissão de Prevenção e Tratamento de Feridas e Núcleo de Segurança do Paciente desta Instituição. Queremos agradecer a cada pt. Acesso em: 15 nov. 2020.

11 Silva JWL, Santos LSA, Caldas GRF, Batista FAM, Sousa MPV, Pereira VCS, et al. Manejo da terapia por pressão negativa (TPN) em lesões complexas. Ebook, Perspectivas contemporâneas das ciências da saúde. Brazilian Journals Editora, v. 3, cap. 22, 2020.

12 Vasconcelos RO, Rigo DFH, Marques LGS, Nicola AL, Tonini NS, Oliveira JLC. Dimensionamento de pessoal de enfermagem hospitalar: estudo com parâmetros oficiais brasileiros de 2004 e 2017. Escola Anna Nery, 2017;21 (4).

13 Silva LC da, Oliveira DAL, Santos ABR, Barbosa LMS, Araújo LG, Barboza MTV, et al. Dimensionamento de pessoal e sua interferência na qualidade do cuidado. Rev enferm UFPE on line. 13(1):491-8, jan. 2019.

um pelo comprimento e dedicação, colocados diariamente nesse trabalho. Todos vocês são a prova de que o sucesso vem através da dedicação e trabalho duro, na imposição de talentos e sonhos. Desta forma, cada um tem contribuído para o nosso crescimento enquanto residentes de enfermagem, permitindo que todos os projetos lançados sejam alcançados. Parabéns a todos pelo trabalho excelente que vem realizando, pelo espírito de união e amor existente a cada um de vocês que acreditaram que seria possível. Compartilhar conhecimento, é o mesmo que disseminar desenvolvimento. Gratidão. 